\title{
Ansiolíticos naturais como alternativa no tratamento da ansiedade
}

\author{
Natural anxiolytics as an alternative in the treatment of anxiety \\ La ansiolítico natural como alternativa en el tratamiento de la ansiedad
}

Recebido: 25/05/2021 | Revisado: 31/05/2021 | Aceito: 22/06/2021 | Publicado: 05/07/2021

\section{Resumo}

Ravena da Silva Portela

ORCID: https://orcid.org/0000-0002-8582-752X Centro Universitário Unifacid, Brasil E-mail: Ravenaportela2012@hotmail.com

Klycia Machado Silva Marques ORCID: https://orcid.org/0000-0001-5419-6350 Centro Universitário Unifacid, Brasil E-mail: klyciamachado@ hotmail.com

Bruna Bezerra Marques ORCID: https://orcid.org/0000-0002-6763-5495 Centro Universitário Unifacid, Brasil

E-mail: bruna_b_marques@hotmail.com

Izaura Maria Rocha

ORCID: https://orcid.org/0000-0002-9023-5852 Centro Universitário Unifacid, Brasil E-mail: Izaura11rocha@hotmail.com

Letice Mendes Ribeiro

ORCID: https://orcid.org/0000-0003-0378-8051 Centro Universitário Unifacid, Brasil E-mail: leticeribeiro@hotmail.com

Ana Flávia Machado de Carvalho ORCID: https://orcid.org/0000-0001-7049-5596 Centro Universitário Unifacid, Brasil

As formas de curar de maneira natural são inúmeras, basta que seja adquirido o conhecimento sobre cada planta e sua verdadeira função. A natureza oferece ao ser humano simples métodos, embora seja preciso cautela e determinação para a obtenção de grandes resultados, uma vez que medicamento manipulados contraindo a dependência, insere o ser humano em um universo que dificilmente compreenderá o verdadeiro sentido de viver em harmonia com a natureza e os ansiolíticos naturais podem ser os grandes heróis para combater a ansiedade, estresse e depressão, grandes vilões existentes em nossas vidas. O objetivo desse estudo é realizar uma revisão integrativa da literatura utilizando artigos científicos e pesquisa de publicações inerentes ao assunto, como artigos e revistas da área farmacêutica. Como critérios de inclusão, caracterizamos artigos originais, completos e gratuitos, publicados entre os anos de 2009 e 2019. Destes, desconsideramos artigos de revisão, teses e dissertações. A estratégia de busca resultou em 12 trabalhos, que foram analisados inicialmente pelos títulos e resumos. Posteriormente, foi realizada uma análise na íntegra dos artigos encontrados, com seleção de 9 artigos que preencheram os critérios de que abordaram o uso de extratos naturais das plantas como estratégia de tratamento para a ansiedade. Apesar de muitos desses ansiolíticos naturais já serem estudadas e utilizadas para este fim, ainda há aspectos relevantes que carecem de estudos. Dessa forma, acredita-se que esse estudo contribuirá para ajudar nos protocolos de tratamento alternativo para pessoas acometidas pela ansiedade, somando-se positivamente com os conteúdos já existentes sobre o assunto.

Palavras-chaves: Ansiedade; Ansiolíticos naturais; Tratamento.

\begin{abstract}
There are innumerable ways of healing in a natural way, just knowing the knowledge about each plant and its true function. Nature offers humans simple methods, although caution and determination are needed to achieve great results, as drug manipulated contracting addiction inserts humans into a universe that will hardly understand the true meaning of living in harmony with nature. Nature and natural anxiolytics can be the great heroes to combat anxiety, stress, and depression, the great villains in our lives. The aim of this study is to perform an integrative literature review using scientific articles and research of publications inherent to the subject, such as articles and magazines in the pharmaceutical area. We found 12 papers that were carefully analyzed by titles and abstracts, but we concluded our research with 9 articles that met the criteria that addressed the use of natural plant extracts as a treatment strategy for anxiety. Although many of these natural anxiolytics are already being studied and used for this purpose, there are still relevant aspects that need further study. Thus, it is believed that this study will help in the alternative treatment protocols for people with anxiety, adding positively with the existing contents on the subject.
\end{abstract}

Keywords: Anxiety; Anxiolytics; Natural; Treatment. 


\begin{abstract}
Resumen
Las formas de curar de forma natural son innumerables, basta con que se adquiera el conocimiento sobre cada planta y su verdadera función. La naturaleza ofrece al ser humano métodos sencillos, aunque se requiere cautela y determinación para obtener grandes resultados, ya que los medicamentos manipulados al contraer dependencia, insertan al ser humano en un universo que difícilmente entenderá el verdadero significado de vivir en armonía con la naturaleza y los ansiolíticos naturales pueden. ser los grandes héroes para combatir la ansiedad, el estrés y la depresión, grandes villanos que existen en nuestras vidas. El objetivo de este estudio es realizar una revisión integradora de la literatura a partir de artículos científicos e investigación de publicaciones inherentes al tema, como artículos farmacéuticos y revistas. Como criterio de inclusión, caracterizamos los artículos originales, completos y gratuitos, publicados entre los años 2009 y 2019. De estos, descartamos artículos de revisión, tesis y disertaciones. La estrategia de búsqueda resultó en 12 artículos, que inicialmente fueron analizados por títulos y resúmenes. Posteriormente, se realizó un análisis completo de los artículos encontrados, con una selección de 9 artículos que cumplían con los criterios que abordaban el uso de extractos naturales de plantas como estrategia de tratamiento de la ansiedad. Si bien muchos de estos ansiolíticos naturales ya han sido estudiados y utilizados para este fin, aún quedan aspectos relevantes por estudiar. Así, se cree que este estudio contribuirá a ayudar con los protocolos de tratamiento alternativo para las personas afectadas por la ansiedad, agregando positivamente al contenido existente sobre el tema.
\end{abstract}

Palabras clave: Ansiedad; Ansiolíticos naturales; Tratamiento.

\title{
1. Introdução
}

A Organização Mundial de Saúde (OMS), em 2002 reconheceu a importância do uso de plantas medicinais para a humanidade classificando a medicina tradicional como: Medicina Complementar, Medicina Alternativa e Medicina Convencional. No sentido de ajudar os países a garantir o uso adequado da Medicina Tradicional e contribuindo para manter a saúde e combater as doenças estabelecendo nesse ano, um Programa de Medicina Tradicional com o objetivo de contribuir com os países e áreas para o desenvolvimento de uso regional e efetivo da medicina tradicional de qualidade.

Souza (2015), destaca que o uso de plantas faz parte da cultura do povo brasileiro e tem sua base na tradição familiar. O conhecimento empírico era e é passado de geração em geração, sem que de fato haja comprovação da eficácia de propriedades medicinais dessas plantas, porém é fato que hoje testes são realizados com mais eficiência e podem apresentar resultados positivos com relação ao uso de plantas medicinais em diversos tratamentos.

A fitoterapia é a utilização de plantas medicinais no tratamento e prevenção de doenças, sendo a forma terapêutica mais antiga. Pode-se ser apresentada de três maneiras: substâncias in natura, manipuladas ou industrializadas. Atualmente, o tratamento com plantas medicinais tem sido muito utilizado pela população, pois a maioria das pessoas procura um tratamento que venha da natureza, que não tenha tanta agressividade no organismo e seja "natural”, porém, muitas pessoas não sabem que há tantos perigos e interações medicamentosas quanto os medicamentos alopáticos (Almeida, 2015).

Faustino, almeida e andreatini (2010), referem-se que as farmacopeias tradicionais apresentam algumas espécies vegetais com indicação para sintomas associados a transtornos de ansiedade. Desse modo, pacientes com transtorno de ansiedade generalizado (TAG) utilizam, entre outros recursos, plantas medicinais, acreditando nas suas inúmeras vantagens. A pesquisa com plantas pode colaborar para a redução da ocorrência de fármacos "me too", que são supostas novidades farmacológicas, mas que, na realidade, são modificações em estruturas já conhecidas e que atuam pelas mesmas vias.

Segundo Lopes (2017), a ansiedade pode ser vista como prejudicial ou até mesmo benéfica ao ser humano, isso é claro, dependerá das circunstancias em que o indivíduo se encontra. Esse transtorno, acompanha o ser humano na sua existência. Numa escala normal da ansiedade, pode ser analisada como um alerta, sinalizando ao indivíduo que ele precisa estar atento pois algo está fora do controle. Já a ansiedade como patologia é caracterizada pela intensidade, pelo sentimento negativo. Ainda com relação a ansiedade patológica ela é desencadeada muitas vezes por doenças.

Dessa forma, Braga, Pordeus e Silva (2019) afirmam que no transtorno de ansiedade ocorre uma ansiedade ininterrupta, que se caracteriza por ter uma duração e intensidade desproporcionais às situações, a qual, além de não ajudar, 
dificulta as reações de defesa ao perigo. Pode vir acompanhada de irritabilidade, tensões musculares, falta de sono, taquicardia, tremores, inquietação, entre outros.

Outro neurotransmissor envolvido com os processos de ansiedade é o ácido gama-aminobutírico (GABA), o principal neurotransmissor inibitório do sistema nervoso central (SNC). Nos transtornos de ansiedade há uma alteração nesses receptore do GABA, estando em número reduzido ou a afinidade pelo neurotransmissor está reduzida. Braga, Pordeus Silva (2019).

Apesar do grande número de estudos pré-clínicos avaliando o potencial efeito ansiolítico de extratos de plantas, poucos estudos clínicos controlados têm sido realizados. Em geral, estes trabalhos apresentam problemas metodológicos (p.ex. pequeno número de pacientes, ausência de grupo controle, ausência de critérios diagnósticos, inclusão de grupos heterogêneos, curto período de tratamento, baixas doses das drogas comparativas) que dificultam uma conclusão mais consistente sobre a eficácia desses extratos. Faustino, Almeida e Andreatini (2010).

Os autores supracitados afirmam que algumas plantas medicinais podem ser utilizadas no tratamento da ansiedade, como a cava-cava (Piper methysticum G. Forst), maracujá (Passiflora incarnata) e a valeriana (Valeriana officinalis). Essas plantas têm ação ansiolítica e atuam no SNC. Faustino, Almeida e Andreatini (2010).

Frente às diversas indagações e incertezas que ainda perduram sobre o tema, objetiva-se nesse estudo analisar os benefícios dos ansiolíticos naturais do tipo passiflora, kava kava e a valeriana como alternativa no tratamento da ansiedade.

\section{Metodologia}

O estudo foi realizado através de uma pesquisa bibliográfica com base descritiva, por meio de revisão de literatura nos indexadores Scielo, PubMed e BVS, utilizando os descritores: ansiedade, ansiolíticos naturais e tratamento. Como critérios de inclusão caracterizamos artigos originais, completo e gratuitos, publicados entre os anos de 2009 e 2019. Foram desconsiderados artigos de revisão, teses e dissertações.

A estratégia de busca resultou em 12 trabalhos que foram analisados inicialmente pelos títulos e resumos. Posteriormente, foi realizada uma análise na íntegra dos artigos encontrados com seleção de 9 após a aplicação dos critérios de inclusão e exclusão.

\section{Resultados e Discussão}

É importante ressaltar que, nos últimos anos, houve um crescente aumento do uso de fitoterápicos no tratamento da ansiedade. O Governo Federal, através de diversas políticas públicas, como a Política Nacional de Plantas Medicinais e Fitoterápicos (PNPMF) e o Sistema Único de Saúde (SUS), tem incentivado a publicação de instruções normativas para ampliar as opções terapêuticas e garantir com segurança e eficácia aos usuários do SUS o acesso a plantas medicinais. (Arruda, 2009).

Dentre elas podemos citar o Maracujá (Passiflora incarnata), a Valeriana (Valeriana officinalis) e a Cava-Cava 5 (Piper methysticum G. Forst). Todas essas plantas têm ação ansiolítica e atuam no Sistema Nervoso Central (SNC), sendo essa última a espécie com o maior número de estudos envolvendo pacientes com transtornos de ansiedade. (Faustino, et al., 2010).

Piper methysticum ou kava-kava encerra nas suas raízes e no rizoma princípios ativos, como as cavapironascom destaque para a cavaína, dihidrocavaína, iangonina, desmetoxi-iangonina, metisticina e dihidrometisticina, às quais respondem pelas propriedades ansiolíticas e antidepressivas, por promoverem efeito relaxante, analgésico e ansiolítico. Comparados aos benzodiazepínicos tradicionais, não causam efeitos colaterais como sonolência e redução da função mental (Toledo, 2015).

As propriedades terapêuticas desta planta têm sido associadas ao tratamento da ansiedade geral e da ansiedade préoperatória, tratamento da insônia e distúrbios de sono, tratamento da síndrome de abstinência, tratamento dos sintomas da 
menopausa, tratamento de cancro, tratamento da hipertensão, tratamento da tosse e no tratamento do distúrbio da hiperatividade e défice de atenção.

Além de indicações terapêuticas, como o tratamento da ansiedade, insônia, irritabilidade, transtornos nervosos e manifestações psicossomáticas, sobretudo em jovens, também é usada no tratamento de sintomas de alcoolismo e da enxaqueca, devido às suas propriedades sedativas. E é uma planta com uso medicinal, a nível mundial (Pereira, 2019).

Por suas propriedades sedativas, hipnóticas e ansiolíticas, a raiz da Valeriana é amplamente usada há várias décadas pela população em geral e médicos, fato que motiva o conhecimento dos benefícios e riscos do uso desta substância. Ela contém uma variedade de compostos químicos incluindo ácido valerênico e derivados, os quais atuam sinergicamente para exercer um efeito sedativo.

O extrato de valeriana mais usado é a Valeriana officinali e tem afinidade para o receptor GABA-A, responsável pela promoção e regularização do sono (Nunes, 2011). O efeito farmacodinâmico da valeriana ajuda a promover e restaurar o sono natural após pelo menos 2-4 semanas de uso. Toledo e Vidal (2015).

A composição química da Valeriana varia de acordo com a subespécie, idade da planta, condições de crescimento, idade e tipo do extrato (Who, 1999). A raiz de Valeriana contém mais de 150 cados, entre eles estão os monoterpenos bicíclicos (valpotriatos componentes já identificados, valtrato e dihidrovaltrato), óleos voláteis (valeranona, valerenal e ácidos valerênicos), sesquiterpenos, lignanas e alcalóides. Também estão presentes aminoácidos livres, como o GABA, tirosina, arginina e glutamina (Galindo, 2011).

A decomposição dos valpotriatos produz pequenas quantidades do ácido isovalérico, que é responsável pelo odor característico desagradável da planta. Essas extrações, são realizadas através da imersão de raiz seca ou rizoma da planta em solução (água, etanol e água ou metanol e água), seguida de centrifugação e secagem para extrair e concentrar os componentes da planta. Segundo a Farmacopéia Americana de Ervas, a extração de ácido valerênico exige pelo menos $30 \%$ de álcool e a extração de valpotriatros exige 70\% de álcool (Oliveira, 2018).

Alguns efeitos colaterais leves foram relatados, como: dor de cabeça, tontura, sonolência, náusea e diarreia. Não foram relatados sintomas hepáticos ou alteração nas funções hepáticas (Nunes, 2011).

Demonstrou-se, ainda, que o dano ao DNA em células epiteliais humanas ocorre somente com doses elevadas. Há poucas evidências que comprovem a interação com medicamentos. Alguns estudos indicaram que não deve ser associado Valeriana com outros sedativos, como antihistamínicos e álcool (Secchi, 2012).

Detectou-se que para comprovar a eficácia do uso de valeriana e avaliar o custo-benefício desta terapêutica nas perturbações do sono e ansiedade, ainda serão necessários vários ensaios clínicos aleatorizados e controlados.

É preciso que haja mais estudos, de melhor qualidade metodológica, que envolvam amostras de dimensão adequada, estandardização das doses e dos tipos de preparação de valeriana usada e que se defina melhor o tempo certo de utilização da mesma para que melhores resultados clínicos sejam obtidos. É importante ainda que as medidas de avaliação de qualidade de sono e ansiedade sejam utilizadas uniformemente entre estudos, para que as conclusões apresentem credibilidade (Nunes, 2011).

\section{Conclusão}

O objetivo desse estudo foi analisar os benefícios dos ansiolíticos naturais do tipo Passiflora, kava kava e Valeriana como alternativa no tratamento da ansiedade. Observou-se que, embora haja um número bem significativo de estudos sobre essas plantas, poucas análises clínicas foram realizadas. Apesar de existir um 6 grande número de estudos que avaliam o potencial efeito ansiolítico dessas plantas, poucas análises clínicas têm sido realizadas. A maioria dos trabalhos que envolvem 
essa temática apresentam problemas metodológicos (p.ex. pequeno número de pacientes, ausência de grupo controle, ausência de critérios diagnósticos, inclusão de grupos heterogêneos, período curto de tratamento ou baixas doses das drogas comparativas). Desse modo, informações valiosas que não tiveram sucesso na coleta de dados acabam dificultando uma conclusão mais consistente sobre a eficácia desses extratos.

\section{Referências}

Almeida, M., Feuerweker, L. C. M., \& Llanos, M. (2015). Educação dos profissionais de saúde na América Latina. Teoria e prática de um movimento em mudança.

Andrade, L. (2001). Aspectos gerais das escalas de avaliação de ansiedade. Rev Psiq Clin, 285-290.

Araújo, P. M. B. (2014). Estresse ocupacional e consumo de ansiolíticos por trabalhadores de enfermagem. Rev enferm. 22 , 615-21.

Arruda, A. M., \& Zanette, V. C., \& Rossato, A. E. (2009). Histórico do uso e aspectos taxonômicos, farmacológicos e toxicológicos. Pesquisa e Extensão em Saúde. 2, 59-68.

Braga, F.F. et al. (2019). Estudo do efeito central do óleo do fruto de Attalea phalerata Mart. ex Spreng, em modelos animais de ansiedade e depressão. Revista Eletrônica de Farmácia.

Carvalho, L. F. (2010). O Modelo De Atenção À Saúde E O Uso De Ansiolíticos Entre Mulheres. Estudos De Psicologia, 9, $121-129$.

Dantas, D. F. C. (2019). Projeto de intervenção para a utilização de terapias alternativas no tratamento dos transtornos de ansiedade, na unidade básica de saúde santa rosa do município de Uberlândia. https://www.nescon.medicina.ufmg.br/biblioteca/imagem.

Faustino, T. T, \& Almeida, R. B, \& Andreatini, R. (2010). Plantas medicinais no tratamento do transtorno de ansiedade generalizada: uma revisão dos estudos clínicos controlados. Revista Brasileira de Psiquiatria.

Galindo, A. S. (2011). Passiflora incarnata 1.: uso terapêutico como ansiolítico natural. Revista Biotemas. 2, 132-148

Gosmann, G. et al. (2011). Composição química e aspectos farmacológicos de espécies de Passiflora L. (Passifloraceae). R. bras. Bioci, 9. 88-99

Lopes, M. W., \& Tiyo, R., \& Arantes, V. P. (2017). Utilização de passiflora incarnata no tratamento da ansiedade. Uningá Review, Paraná, $29,81-86$. Nunes, A.; Sousa, M. (2011). Utilização da valeriana nas perturbações de ansiedade e do sono. Acta Med Port.v. 24, $961-966$.

Oliveira, Y. R. (2018). Ansiedade: aspectos gerais e tratamento com enfoque nas plantas com potencial ansiolítico. Revinter, 11, 33-54

Pereira, S. M.T. (2019). O uso medicinal da profilaxia incarnata L. http://bdm.ufmt.br/handle/1/1251.

Santana, G. S., \& Silva, A. M. (2019). O uso de plantas medicinais no tratamento da ansiedade. III simpósio de assistência Farmacêutica.

Secchi, P.; Virtuoso, S. (2012). O efeito da valeriana no tratamento da insônia. Visão Acadêmica. 13

Silva, J. D. T. et al. (2010). Kava-kava como aditivo fitoterápico na alimentação de codornas de postura. Revista Biotemas, 23

Soldatelli, M. V.; Ruschel, K., \& Isolan, T. M. P. (2010). Valeriana offi cinalis: uma alternativa para o controle da ansiedade odontológica? Stomatos, 89-9.

Sousa, F. C. F. et al. (2009). Plantas medicinais e seus constituintes bioativos: Uma revisão da bioatividade e potenciais benefícios nos distúrbios da ansiedade em modelos animais. Revista Brasileira de Farmacognosia. 18, 642-654

Souza, M. F. B. (2015) Plantas medicinais com potencial terapêutico ansiolítico no Brasil: uma Revisão Integrativa. http://bdm.ufmt.br/handle/1/1251.

Toledo, C., \& vidal, r. (2015). Valeriana officinali 1. No tratamento da insonia e ansiedade. Brazilian Journal of Surgery and Clinical Research, 9, 78-83.

Torchi, C. Belizario, Gueda, S., \& Silva, A. M. (2014). Seguimento do uso da passiflora incarnata no tratamento da insônia. II Simpósio de assistência farmacêutica. 